\title{
Engineering Logical Inflammation Sensing Circuit for Gut Modulation
}

\author{
Liana N. Merk ${ }^{1, *}$, Andrey S. Shur ${ }^{1}$, Ayush Pandey $^{2}$, Richard M. Murray $^{1,2}$, and Leopold N. Green ${ }^{1}$ \\ ${ }^{1}$ Department of Biology and Biological Engineering \\ ${ }^{2}$ Department of Control and Dynamical Systems \\ California Institute of Technology, Pasadena, CA \\ *Corresponding author email: lmerk@caltech.edu
}

\begin{abstract}
The mammalian gut contains trillions of microbes that interact with host cells and monitor changes in the environment. Opportunistic pathogens exploit environmental conditions to stimulate their growth and virulence, leading to a resurgence of chronic disorders such as inflammatory bowel disease (IBD). Current therapies are effective in less than $30 \%$ of patients due to the lack of adherence to prescription schedules and overall, off-target effects. Smart microbial therapeutics can be engineered to colonize the gut, providing in situ surveillance and conditional disease modulation. However, many current engineered microbes can only respond to single gut environmental factors, limiting their effectiveness. In this work, we implement the previously characterized split activator AND logic gate in the probiotic E. coli strain Nissle 1917. Our system can respond to two input signals: the inflammatory biomarker tetrathionate and a second input signal, IPTG. We report 4-6 fold induction with minimal leak when both signals are present. We model the dynamics of the AND gate using chemical reaction networks, and by tuning parameters in silico, we identified perturbations that affect our circuit's selectivity. We anticipate that our results will prove useful for designing living therapeutics for spatial targeting and signal processing in complex environments.
\end{abstract}

\section{Introduction}

Approximately $10^{13}-10^{14}$ bacterial cells live in the dynamic and complex community within the gut microbiome, where they can impact numerous facets of human health [1-3]. The idea that changes in the composition of this community result in disease offers a compelling motivation for engineering microbes to sense, cure, or prevent these perturbations. Engineering microbes for gut diagnostics and therapeutics is a growing field in synthetic biology, owing to the tractability and relative safety of genome engineering in microbes. Recent examples include inhibition of pathogenic Pseudomonas aeruginosa via antimicrobial peptides [4] and delivery of checkpoint blockade nanobodies to tumors [5]. Due to the presence of bacteria in a wide variety of ecological niches, there exists a wide variety of evolved sensors for therapeuticallyrelevant molecules [6]. Microbes' ability to sense and respond to stimuli in situ offers controlled and targeted responses to traditionally difficult locations of disease.

One such complex microbiome perturbation is inflammation, which can drive dysbiosis, or disruptions in microbial populations [7]. Dysbiosis is a root cause of inflammatory bowel disease (IBD) and has been associated with infectious disease, obesity, and other medical disorders [8]. During IBD, specific niches within the gut microbiome change, causing spatial heterogeneity [9]. Currently, the most common treatment method for IBD is large doses of oral anti-inflammatory drugs, which have broad and nonspecific effects that do not account for the local environmental changes [10]. These anti-inflammatory drugs often require irregular and frequent dosage schedules challenging to adhere to, with the average patient missing half of their treatments [11].

Microbes capable of drug manufacture and secretion can colonize the microbiome, providing long-lasting in situ therapeutics without complex dosing schedules. Recent studies have found promise for inflammation treatment with microbes secreting interleukin-10 (IL-10) [12], IL-27 [13], and anti-tumor necrosis factor $\alpha[14]$. However, these microbes express target molecules constitutively, not taking advantage of the ability 


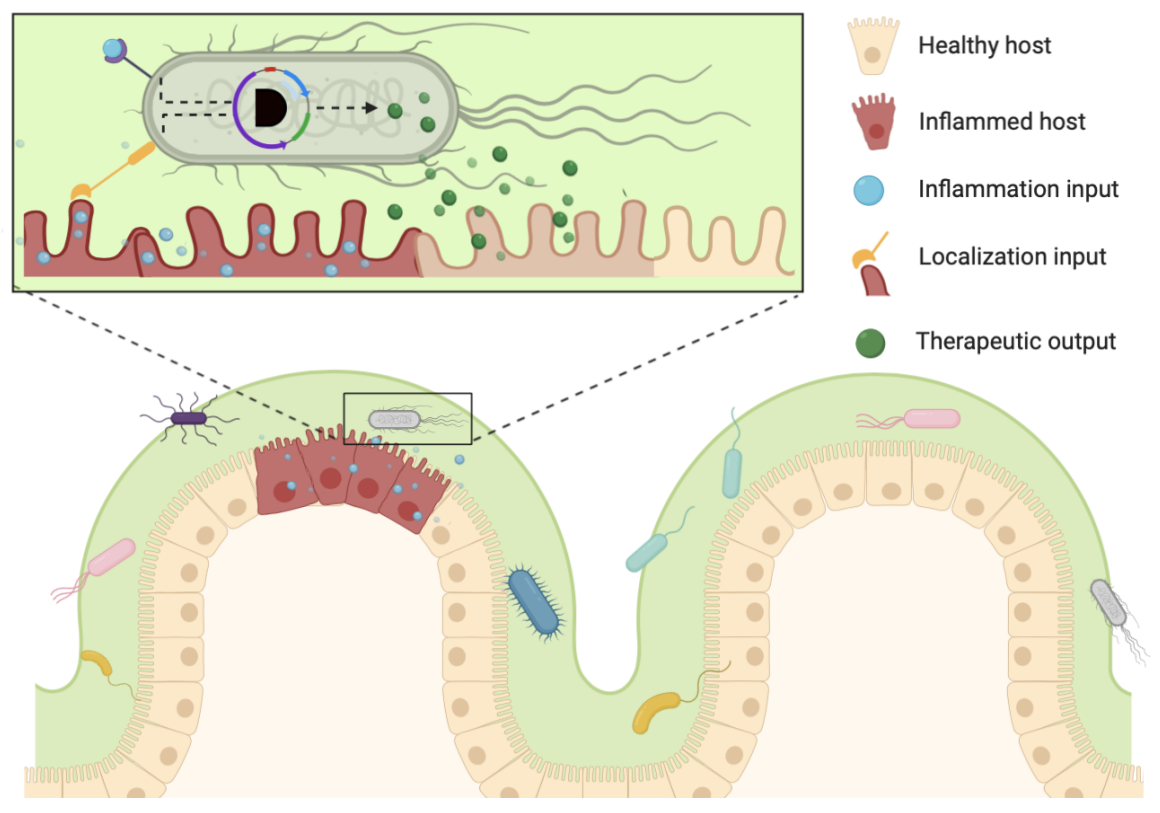

Figure 1: An example of how AND gates would provide spatial targeting. Production of the anti-inflammatory drug will only be active if cells detect that they are localized to the gut epithelium and the inflammation biomarker tetrathionate is present. Thus, anti-inflammatory action is only performed if the therapeutic cells are in the right place, minimizing off target effects. Figure not drawn to scale.

of bacteria to sense and respond to their environment. This is especially relevant in IBD, a disease known to have spatial heterogeneity [7].

Genetic circuits responsive to inflammation are thus of great interest to the field of microbiome engineering. Two inflammatory biomarkers, nitric oxide [15] and tetrathionate [16] have been used as triggers for memory circuits, allowing for in situ inflammation diagnosis. The second biomarker, tetrathionate, has also been used to drive expression of microcins capable of inhibiting Salmonella spp. outgrowth [17]. Tetrathionate was first noted as a biomarker of intestinal inflammation by Winter et al. [18]. Salmonella Typhimurium was found to cause acute gut inflammation, causing the release of reactive oxygen species. These species react with intestinal sulfur compounds, most notably thiosulfates, causing the formation of a new respiratory electron acceptor, tetrathionate. S. Typhimurium and other pathogens, like C. jejuni, can gain a growth advantage by using tetrathionate as an electron acceptor.

Daeffler et al. [6] identified a tetrathionate two-component sensor from the marine bacterium Shewanella baltica OS195. The system consists of a membrane-bound sensor histidine kinase, ttrS, and a cytoplasmic response regulator, ttrR. Tetrathionate binds to ttrS, causing phosphorylation, leading to a complex that can phosphorylate ttrR. Phosphorylated ttrR, in turn, activates pTtr, which demonstrates low cross-activation by a range of other ligands.

We implemented the hypersensitive response and pathogenicity (hrp) system reported by Wang et al. [19] to incorporate logical sensing. This system consists of two co-activating genes hrpR and hrpS, which activate the $\sigma 54$-dependent hrpL promoter [20]. The genetic elements were isolated from Pseudomonas syringae and optimized to function with various synthetic biology promoters, including pLac. Because the circuit is orthogonal to the $E$. coli genetic background, it can be engineered into $E$. coli Nissle without host genome interference. Here, we unite the inflammatory relevance of tetrathionate sensing with the regulatory control offered by this split activator system.

While microbes have been engineered to respond to environmental factors like inflammation, they are often limited to sensing single inputs. Here, we develop a logical microbiome response circuit that produces an output only in the presence of inflammation and an external activator. We build upon the previously char- 
acterized split activator AND logic gate to show selective activation to inflammatory biomarker tetrathionate and isopropylthiogalactoside (IPTG). We develop a mathematical model of the tetrathionate two-component system to validate the experimental optimization steps, shown in Section 2.1. Then, in Section 2.2, we describe experimental results to optimize the two-component system in E. coli Nissle. Building on this further, we present our main result in Section 2.3. We develop a two plasmid construct that responds selectively to IPTG and tetrathionate. We conclude our results in Section 2.4 with a discussion on the design space exploration for the AND gate circuit using a detailed model that describes the effects of leak and points towards potential improvements in the circuit design. Our work expounds on prior work establishing a new class of microbial-based therapeutics that processes two inputs to respond in a targeted manner in complex environments.
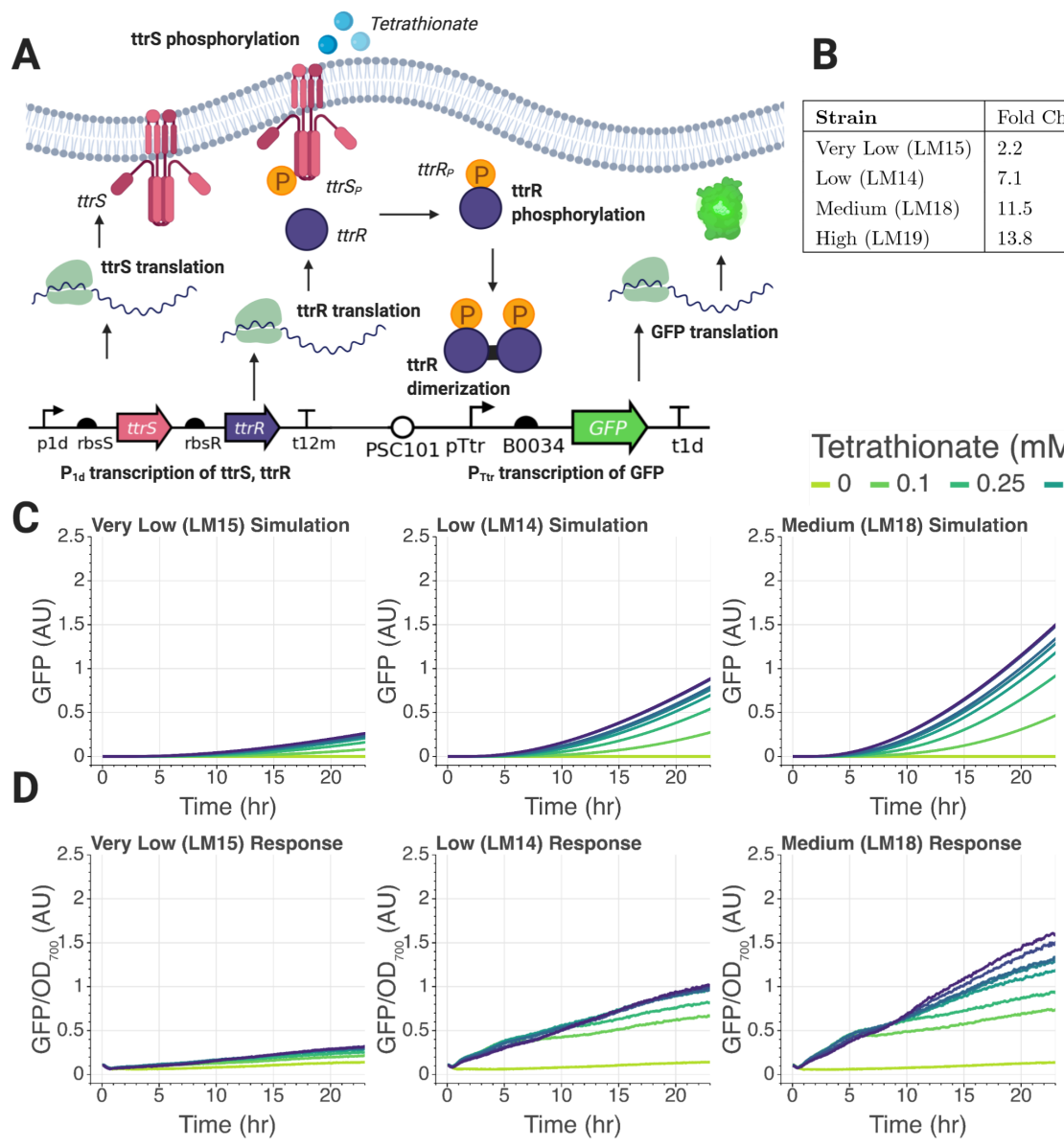

\begin{tabular}{|c|c|c|c|c|c|}
\hline & \multicolumn{2}{|c|}{ Calculated } & \multicolumn{2}{|c|}{$\mathrm{k}_{\text {off }} \frac{1}{s}$} \\
\hline Strain & Fold Change & rbsS & rbsR & rbsS & rbsR \\
\hline Very Low (LM15) & 2.2 & 23 & 42 & 20 & 15 \\
\hline Low (LM14) & 7.1 & 15 & 23 & 12 & 8 \\
\hline Medium (LM18) & 11.5 & 13 & 132 & 11 & 6 \\
\hline High (LM19) & 13.8 & 93 & 47 & 10 & 5 \\
\hline
\end{tabular}
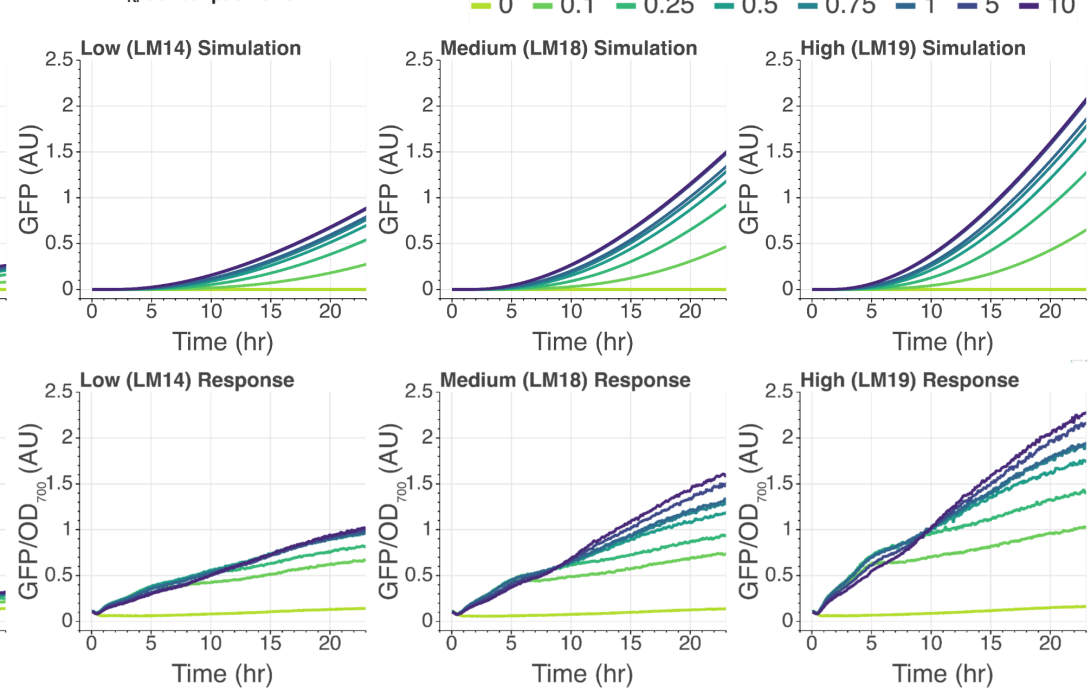

Figure 2: Expression and Simulation of Tetrathionate Response Circuit. A. Schematic of tetrathionate two component system. All shown steps were modeled using chemical reaction networks. B. Relative RBS binding strengths to ttrS and ttrR calculated from sequenced RBS sites isolated from 4 functioning tetrathionate response circuits. Off rates for simulated reactions. C. Simulations of RBS tuning by varying ribosome off rates to each ribosome binding sites. D. Plate reader time course with increasing tetrathionate concentrations and different ribosome binding strengths. 


\section{Results}

\subsection{Modeling the Tetrathionate Two-component System}

To validate the experimental optimization steps, we use simulations of a mathematical model that we built by writing the chemical reactions that describe the dynamics of the two-component system. Two component signaling is one of the most prevalent methods that microbes gene expression from transmembrane signals [21]. To understand the effects of various experimental tuning steps such as the ribosome binding strength on the expected output, we describe a model for the tetrathionate signaling system. This model is divided into three parts - the expression of tetrathionate regulators ttrS and ttrR, phosphorylation of the tetrathionate regulators, and the reporter gene's activation.

The regulator's constitutive expression, ttrS and ttrR are under the common promoter J23103 (P1d) [22]. Since these parts are co-transcribed, we model a single transcription reaction for both regulators. Transcription is modeled as a two-step process: binding the promoter to the RNA polymerase to form a complex and transcription of the complex to the corresponding mRNAs. Since each regulator is under control of its own ribosome binding site, we have modeled two separate translation reactions. The translation is also modeled as a two-step process where a ribosome binds to the mRNA transcript to form a complex that then translates to express the regulator protein.

For the phosphorylation pathway, we model the tetrathionate molecule binding to ttrS as reversibly triggering phosphorylation. The cytoplasmic response protein ttrR can bind to either the phosphorylated or dephosphorylated ttrS. If ttrR binds to dephosphorylated ttrS, there is a higher OFF rate than if trR binds to phosphorylated ttrS. Phosphorylation of ttrR only occurs once it binds to the phosphorylated ttrS forming a complex, $\operatorname{trR}: \operatorname{ttr} \mathrm{S}^{\mathrm{P}}$. It is not known whether the dephosphorylation of $\operatorname{ttrR}$ is phosphatase dependent, like the KdpD/KdpE TCS in E. coli [23]. Here, we do not model phosphatase explicitly but rather set an explicit rate, $k_{\text {dephos }}$, that defines the dephosphorylation of $\operatorname{ttrR}$.

The third and final part of the two-component signaling model is the activation of the GFP reporter gene. Phosphorylated ttrR may dimerize reversibly within the cytoplasm. It is not known whether inactive ttrR (that is, dephosphorylated dimers or monomers) can bind to the promoter region. Here, we only model the dimerized, phosphorylated ttrR as activators of gene expression. Once the activated ttrR binds, the inactive pTtr promoter is converted to an active state, denoted as $\mathrm{pTtr}^{*}$. RNA polymerase may only bind to this activated promoter. This triggers the transcription of the GFP transcript, GFP $_{\mathrm{T}}$. This transcript then binds reversibly with a ribosome and is irreversibly converted into the unbound ribosome, $\mathrm{GFP}_{\mathrm{T}}$, and GFP protein.

Choosing reaction rate parameter values for the different chemical reactions is a critical step in modeling the signaling system. Since finding exact values for all the rate parameters apriori is impossible, we make a few simplifying assumptions. We assume that the explicit translation rates are identical for all mRNAs in the system. Further, we also assume that all degradation reactions occur at the same rate. We selected the nominal values from the various results and information available in the literature, discussed further in the supplementary information for all parameters. Since the ribosome binding strength is a tunable parameter in the experimental design, we keep this parameter free to change during the simulations to observe its effects. The different RBS strengths are modeled by changing the ribosome's unbinding reaction rates to a given transcript.

The model simulations with varying ribosome binding strengths are shown in Figure 2-C. The model predicts that the RBS preceding the inducers has a strong effect on output fold change. With increasing ribosome binding strengths, the model simulations show an increased fold change and higher reporter protein expression. The experimental results seem to show a similar performance with changing RBS strength, as shown in Figure 2-D. We used a Python toolbox called BioCRNpyler [24] to generate the model and ran simulations using the Bioscrape [25] simulator. The detailed model including the system's species, reactions, and parameters is given in the supplemental information. The Python code used to generate the simulations shown in Figure 2-C is available publicly on Github [26].

A future line of work would be to quantitatively validate the model parameters by fitting the experimental data to the simulations so that the model can be used to make credible predictions. Since various parameters in the model are context-dependent, parameter tuning of a validated model in silico may provide helpful insights when implementing this circuit in the gut environment. Similarly, the effects of resource 
sharing and high burden due to the expression of proteins may be quantified using this model. Towards that end, we discuss the results from preliminary data collected for the TCS in the next section.

\subsection{Tetrathionate sensor validation}

The end goal of our construct is implementation into the microbiome. As such, we wanted to minimize the number of plasmid constructs present in our system. We designed a single construct to have all regulators as well as the tetrathionate inducible promoter, pTtr, shown in Figure 2-A. We engineered the inducible promoter after the regulators, which are constitutively expressed. Thus, if the polymerase reads through the regulator terminator, it would not express the pTtr construct.

Using 3G assembly [27], we constructed a tetrathionate-responsive circuit. To minimize leak while maximizing circuit response, we screened regulators of varying expression strengths. We optimized the RBS preceding both tetrathionate sensing regulators ttrS and ttrR using the Andersen RBS pool [22]. We transformed constructs into JM109 E. coli strain and selected six constructs for sequencing. We estimated the RBS strengths of the sequenced circuit constructs shown in Figure 3-B [28]. Weak RBS preceding ttrS or ttrR leads to a lower fold change of GFP expression upon the addition of tetrathionate, as seen in the simulated results as well. The high activation circuit, LM19, was selected for the next steps due to $13.8 \mathrm{x}$ activation with maximum tetrathionate input.

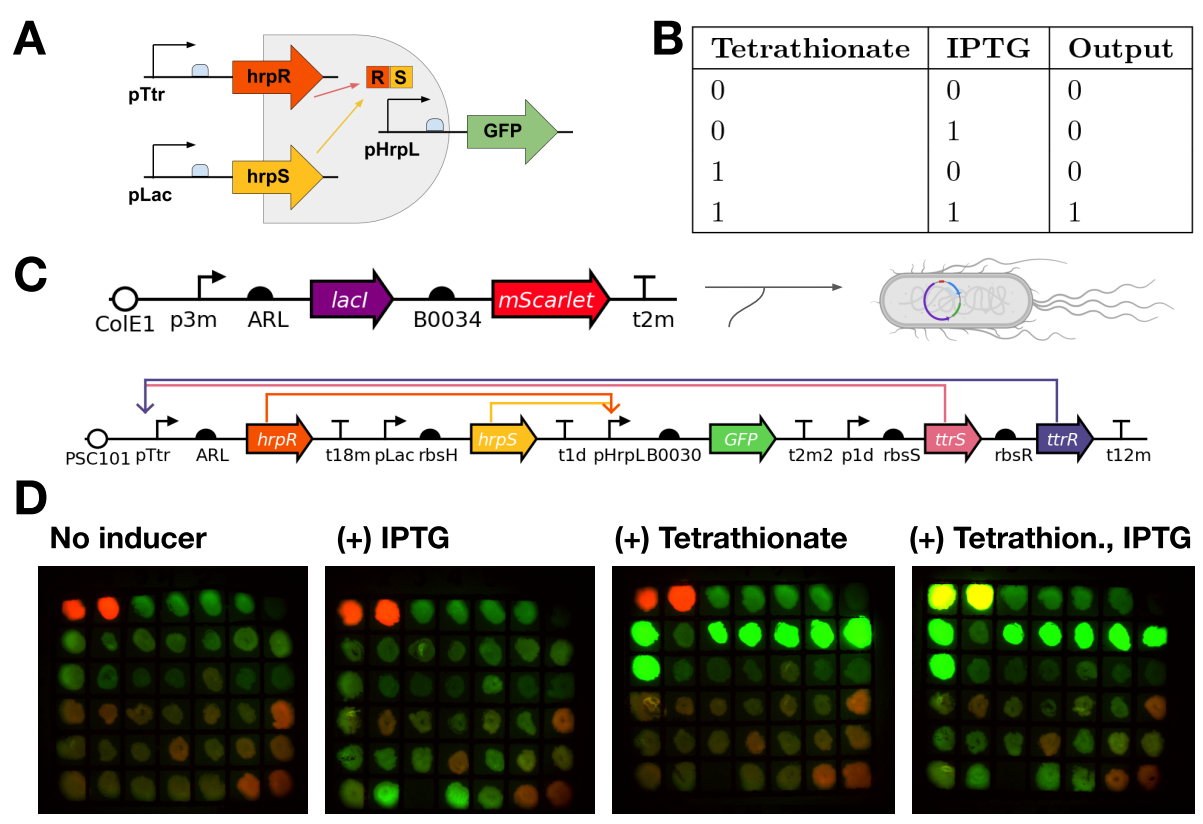

Figure 3: AND Gate Design and Screening. A. The $\sigma 54$-dependent promoter hrpL is activated by two activators: hrpR and hrpS. The expression of hrpR is driven by pTtr, the tetrathionate response promoter. The second activator, hrpS, is driven by pLac which is induced by IPTG. Should the AND gate function properly, we expect GFP expression only when both inducers are present. B. A truth table displaying the expected output. C. The circuit diagram for co-transformed plasmids. The PSC101 backbone contains KanR, conferring kanamycin resistance. The ColE1 backbone contains ChlorR, conferring chloramphenicol resistance. D. Plate screening of AND gate constructs. LB plates contained max inductions of IPTG $(1 \mathrm{mM})$, Tetrathionate $(1 \mathrm{mM})$, neither, or both. Colonies were streaked on all four plates. Successful colonies were red fluorescent in all plates, signifying constitutive mScarlet production, and green fluorescent on the double inducer plate, signifying AND activation. Colonies in the top left two positions were miniprepped and re-transformed into Nissle. 


\subsection{Engineering two-input AND Gate}

To incorporate logical sensing, we chose a system of co-dependent split activators to drive expression of our reporter gene. One regulator, hrpR, is driven by the tetrathionate-dependent promoter. The second regulator, hrpS, is driven by pLac, which activates in the presence of IPTG. These two regulators form a homo-hexameric complex needed to activate $\mathrm{pHrpL}$, which drives GFP expression, shown in Figure 3-A. Our circuit should thus only activate in the presence of tetrathionate and IPTG, shown in the truth table in Figure 3-B.

While this split activator system has previously been tested in seven different chassis [19], it has not been tested in E. coli Nissle 1917. We first performed one round of optimization of the AND gate components in E. coli Marionette Clo cells [29] which natively expresses lacI. Initially, the RBS reported by Wang et al. [19] were used, with B0034 for hrpR and rbsH for hrpS. However, expression was leaky in all inducer conditions, suggesting that the activator expression might be too high. To resolve this, we cloned the Anderson library of ribosome binding sites (ARL) [22] in front of hrpR. We then isolated five constructs with significant fold change in response to both inducers - IPTG and tetrathionate. Constructs were isolated, sequence verified, and re-transformed into Nissle, shown in Figure 3-D.
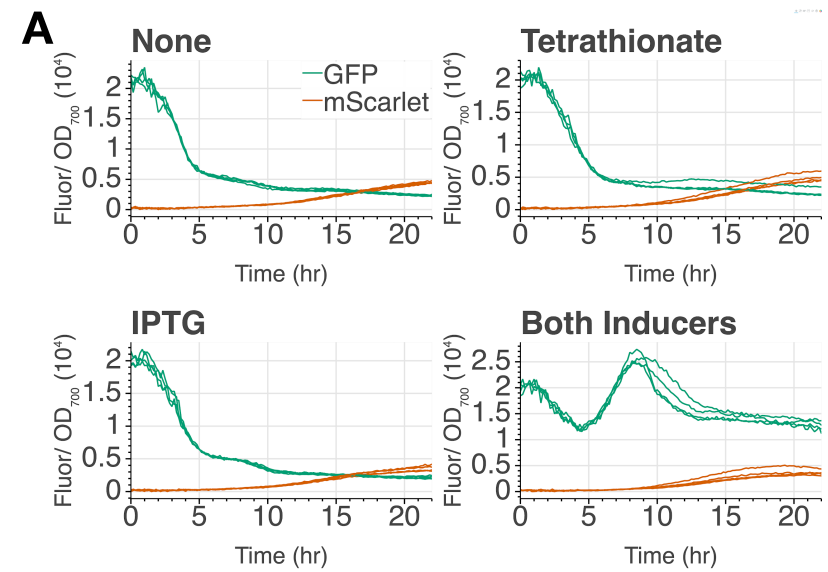

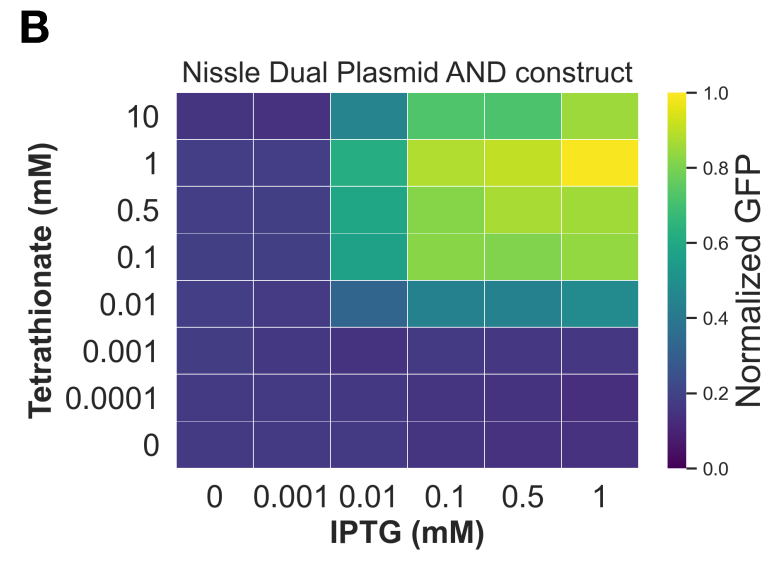

Figure 4: AND Gate Experimental Results. A. Plate reader assay for Nissle AND constructs. Maximum induction of $1 \mathrm{mM}$ tetrathionate and $1 \mathrm{mM}$ IPTG was used. Fluorescence values were normalized to OD700 readings. At the experiment's onset, low OD causes high normalized values. Maximum induction is achieved by hour 8. B. Heatmap of increasing inducer concentrations. Values displayed are GFP fluorescence normalized to OD, divided by the maximal value, which was achieved at $1 \mathrm{mM}$ IPTG and $1 \mathrm{mM}$ tetrathionate. To test the upper bound of tetrathionate sensing, $10 \mathrm{mM}$ was used, showing slight decrease from the $1 \mathrm{mM}$ condition. This could be caused by resource loading or cell toxicity at this concentration.

To optimize this system in E. coli Nissle, we found proper expression level of lacI such that pLac can be regulated. We co-transformed a constitutively expressed lacI and mScarlet plasmid with the working AND gate plasmid optimized in Marionette Clo. Using stereoscope screening and plate reader assays, we confirmed the selective AND gate functionality of one dual-plasmid construct, shown in Figure 4-A. In our experimental results, we observed a 6-fold induction once tetrathionate and IPTG are both added and minimal expression in the one or no inducer conditions. We further show the AND gate displays digital-like activation across a range of both inputs Figure 4-B. To the best of our knowledge, this is the first functioning inflammatory AND gate in E. coli Nissle.

\subsection{Design space exploration with an AND gate model}

Like the two-component system model, we modeled the AND gate circuit by describing all of the different mechanisms and components discussed in the previous section. With this model, we explore two key design 
aspects of the system - the effect of RNA polymerase binding selectively with the combinatorial promoter in the presence of both activator proteins and the effect of initial conditions of the LacI repressors. This design space exploration points towards further design optimizations possible in the circuit, which would be essential to harness when implementing this AND gate in the gut microbiome.

\section{Effects of RNAP binding}

As shown in Figures 5-A and 5-B, RNA polymerase can bind to the combinatorial promoter, pHrpL, either when both the activators hrpR and hrpS are present, or when only one of those is bound to the promoter. Of course, this binding specificity is not experimentally tunable and depends on the designed constructs and chassis. To study all of these possible interactions and their effects, we model the corresponding reactions in a coarse-grained model. We model the logic gate circuit with a non-zero amount of both split activator proteins, hrpR and hrpS (R and S, respectively). Using this logic gate model, we performed preliminary parameter searches to study the combinatorial promoter dynamics.

Due to their high sequence similarity, it may be possible for a single hrp activator (R or S) to gain the ability to activate transcription, perhaps through mutations in binding domains. To account for this

A
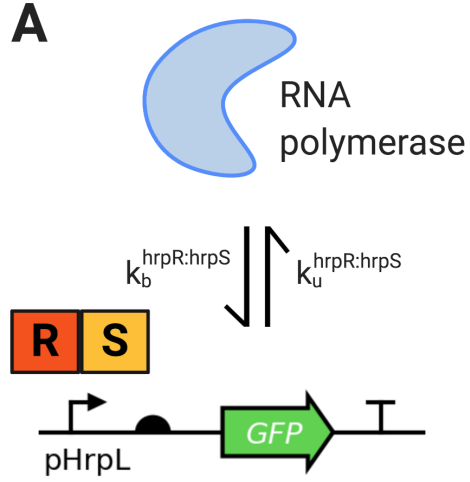

B

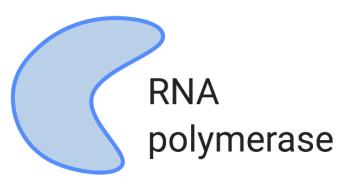

R

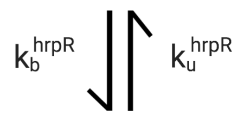

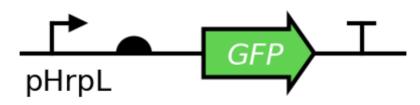
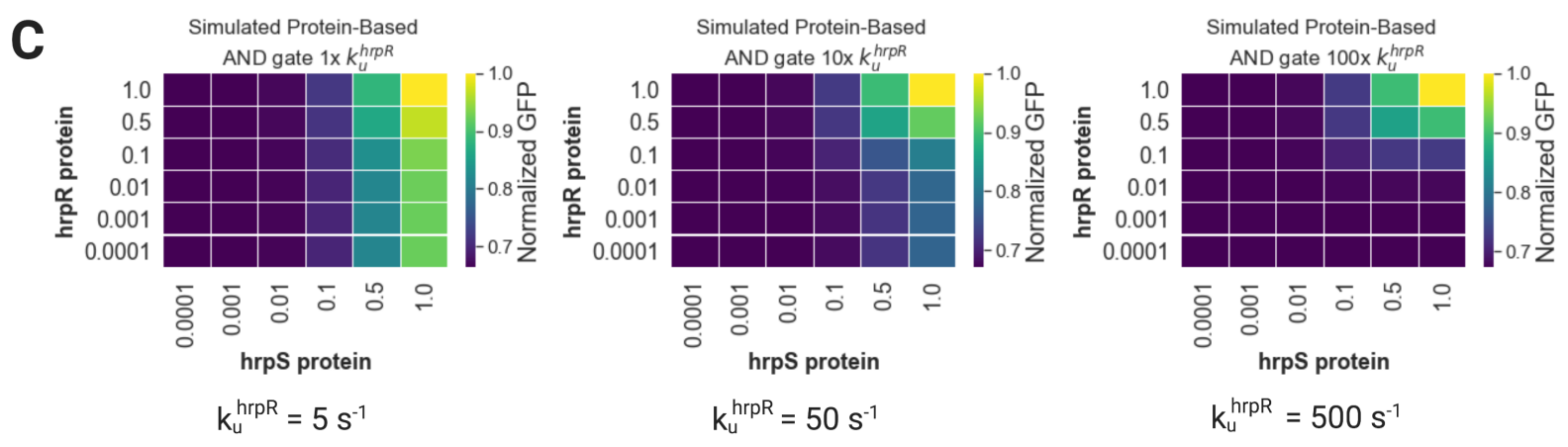

Figure 5: Parameter Tuning in Protein AND Gate Model. A. Both regulators may bind to the promoter region, creating a complex capable of being transcribed. RNA polymerase binds and unbinds with the rates shown. B. A single activator is also able to trigger transcription. Here, we show the effects of tuning hrpR's ability to trigger transcription. C. Examples of how decreasing off-rate of RNAP to the pHrpL:hrpRaffects the output. We see significant loss of reliance on the second activator, hrpS, when hrpR is allowed to form an active transcription complex that unbinds less frequently. Note, this result is symmetric. If we model hrpS, the AND gate functionality changes in ways consistent to those shown here. 
case, we allow a single activator-DNA-RNA polymerase complex to activate transcription at a decreased rate relative to the two activator complex. In Figure 5-C, we simulate this effect by varying the unbinding rate of RNAP to the pHrpL:hrpR complex by more than 100 -fold. We begin with a very low unbinding rate, signifying that RNAP is able to transcribe the single activator complex, pHrpL:hrpR, more readily. Indeed, we see that even small amounts of hrpR are now sufficient to activate the AND gate. The circuit still activates readily at high hrpS, since the $k_{u}^{h r p R: h r p S}<k_{u}^{h r p R}$, conferring a preference for the double activator case. Increasing the $k_{u}^{h r p R} 100$-fold in Figure 5-C results in AND gate functionality. This implies that the AND gate's functionality depends on the RNA polymerase's binding specificity to the combinatorial promoter when both activating signals are present. The experimental result for this AND gate implemented in Nissle, as shown in Figure 4, displays similar performance as in the model simulations in the last panel of Figure 5-C. Hence, we can hypothesize about this unknown mechanism of RNAP binding and conclude that it is indeed a specific binding that occurs primarily when both activators are present.

To further analyze the system behavior we expanded the coarse-grained model of the AND gate to include the expression of the two activators, hrpR and hrpS as well. We established earlier that the promoter can initiate transcription when both activators are bound, but also at a slower rate if one or neither activator binds. The detailed model consisting of all the transcription and translation reactions of this construct also confirms this. In addition, using the detailed model, we can simulate and understand the dynamics of the activator transcript and protein levels as well as the dynamics of the reporter transcript and its expression level. We developed this detailed model using BioCRNpyler [24] and for all model simulations, we used parameters and initial conditions consistent with those found in the E. coli literature [30].
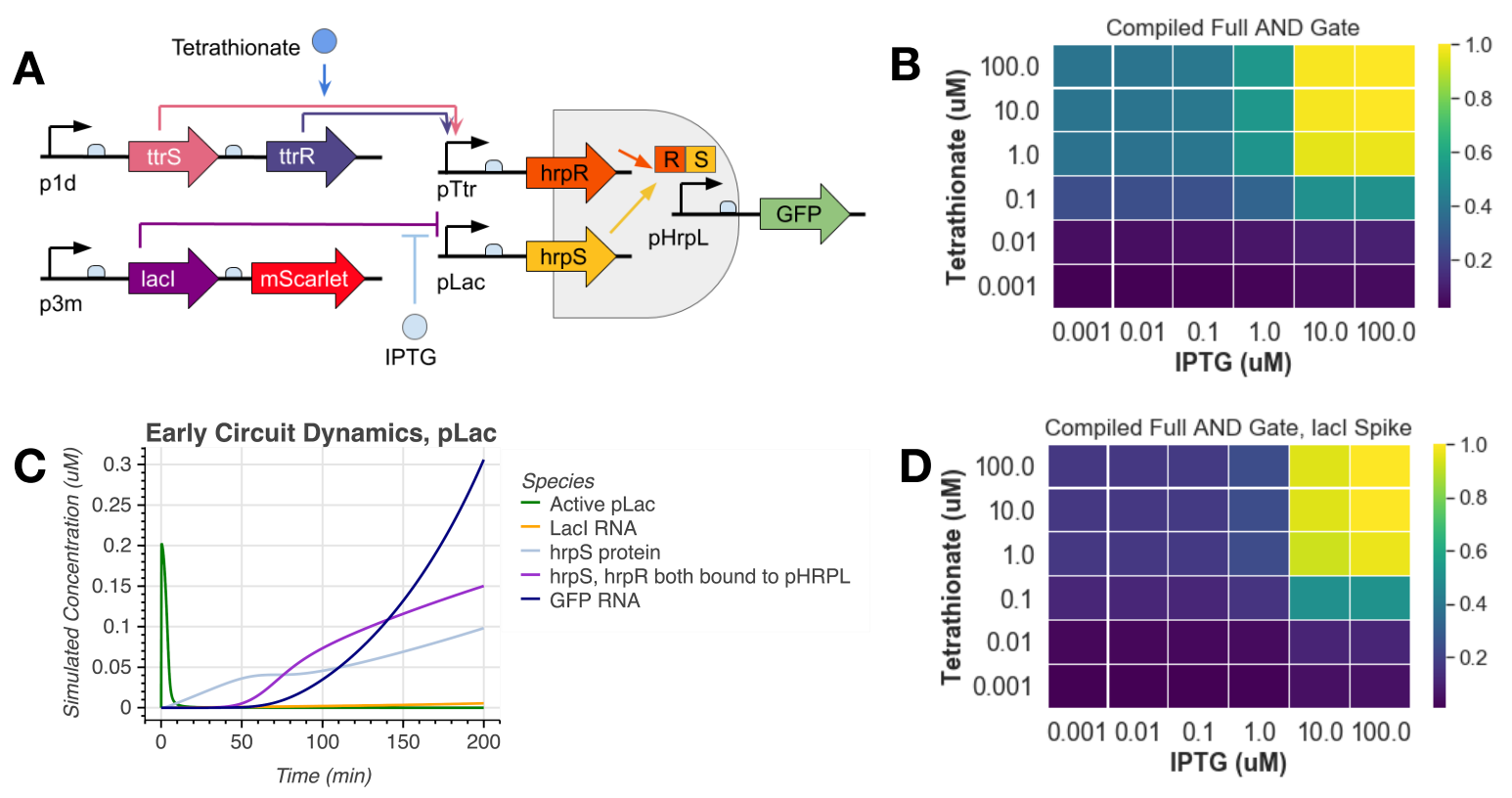

Figure 6: Full AND Gate Model. A. We model expression of the two split activator components. This detailed model allows us to tune various parameters to better understand dynamics. B. In the simulated heatmap, we see leak along the IPTG axis, telling us there is some underlying activation of pLac even when IPTG concentrations are below the activation threshold. C. When we input $0 \mathrm{mM}$ IPTG and $1 \mathrm{mM}$ tetrathionate, we see that there is still a burst of active pLac in the beginning of the time course. This burst of hrpS expression led to increasing amounts of GFP throughout the simulation. D. This led us to spike in $1 \mathrm{mM}$ lacI at the beginning of the experiment, preventing the initial spike of unrepressed pLac. 


\section{Effects of repressor initial conditions}

To study the effect of initial conditions for the LacI repressor, we use the LacI repression mechanism that we modeled in our full system description. Two LacI molecules bind to DNA forming a repressed complex that can not be transcribed. A single IPTG molecule can sequester LacI in solution, preventing the repressor complex from binding to DNA. IPTG can also bind to the LacI complex bound to DNA, releasing LacI from the operator region. The AND gate activator hrpR is under the expression of the pLac promoter. The circuit schematic corresponding to the model is shown in Figure 6-A. Note that when LacI is not bound to pLac, hrpR is expressed constitutively. We explore the model simulations by plotting the steady state GFP values under different input conditions as shown in the heatmaps in Figure 6-BD.

A key design aspect that results from this analysis is that if we start with no LacI present, we obtain leaky activation of the AND gate independent of the IPTG levels as shown in Figure 6-B. This is counterintuitive since LacI is constitutively expressed in the system. A possible hypothesis could be that the delay in transcription of LacI leaves enough time for hrpR expression, even at low levels, hence giving a leaky reporter expression. By looking at the time-course dynamics of the system carefully, we find that this is indeed the case. We observe that there is a sharp spike in actively transcribing pLac. This causes small amounts of hrpR transcription, which is enough to activate the AND gate. It is important to note that increasing the rate of LacI transcription/translation and decreasing the rate of pLac transcription does not fix this issue. However, we know that the engineered microbe is such that it will always have cellular LacI diluted in from its parent. So, the case study with zero initial condition for LacI is not applicable in practice. To account for this in our model, we simply spiked low concentrations of LacI as an initial condition, thus resulting in recapitulation of the expected AND gate dynamics as shown in Figure 6-D.

\section{Methods}

\subsection{Plasmid construction and strains}

Plasmid maps and screening methods are provided in Supplementary Figure 8. All constructs were assembled using 3G assembly, as described [27]. Constructs were sequence verified by sequencing (Laragen) after amplification with UNS primers. The sequence for ttrS was identified from Addgene PKD227. We obtained sequences for ttrR, and pTTR sequences from Addgene plasmids pKD233.7-3. gBlocks with these sequences were ordered from Twist Biosciences and resuspended in IDTE buffer. Logic gate parts were gifts from Martin Buck \& Baojun Wan. hrpS was amplified from pBW213 (Addgene 61435) hrpR was amplified from pBW115 (Addgene 61434), pHrpL was amplified from pBW412hrpL-cIgfp (Addgene 61438). The BSAI cut site was removed from hrpS by Gibson assembly. B0030 and RBSH sequences were obtained from [19] and synthesized by IDT.

Tetrathionate regulator optimization circuits were transformed into chemically competent JM109 (Zymo Research). Optimization of AND gate constructs was performed by making constructs with ribosome binding site library (5'-GAAAGANNNGANNNACTA-3') in front of regulators in chemically competent Marionette Clo cells prepared from Addgene [29]. Plasmids were miniprepped and re-transformed into electrocompetent Nissle 1917 (Mutaflor). Antibiotic concentrations used in all growth was $34 \mathrm{\mu g} / \mathrm{mL}$ chloramphenicol and 50 $\mu \mathrm{g} / \mathrm{mL}$ kanamycin.

\section{$3.2 \quad$ In vitro aerobic experiments}

Colonies were screened using stereoscope images of LB agar inducer plates with $1 \mathrm{mM}$ potassium tetrathionate (Sigma Aldrich), 1 mM Isopropyl-beta-D-thiogalactoside (Sigma Aldrich), or both. Successful colonies that were only fluorescent in the presence of both inducers were then used for in vitro screening.

These colonies were grown overnight in M9CA media (Teknova) to saturation. Cultures were then diluted 1:5 and grown for three hours. Characterization was performed in 96 well Matriplates (Dot Scientific, MGB096-1-1-LG-L). Where applicable, inducer media was prepared in M9CA and used to dilute outgrown cells. Plates were incubated at $37^{\circ} \mathrm{C}$ for 23 hours in a Biotek Synergy H2 plate reader with continuous shaking at $282 \mathrm{cpm}$. Optical densities (OD700) and fluorescence measurements were taken every 5 minutes from the bottom of the plate. GFP excitation and emission wavelengths were $483 \mathrm{~nm}$ and $510 \mathrm{~nm}$, respectively. 
mScarlet excitation and emission wavelengths were $565 \mathrm{~nm}$ and $595 \mathrm{~nm}$, respectively. Gain 100 was used for both fluorescence channels.

\section{Discussion}

This work demonstrates the engineering and modeling of a two-input AND gate for downstream therapeutic applications, where one input is the medically relevant inflammation signal tetrathionate. We optimized our circuit in E. coli Nissle, an attractive microbe for microbiome engineering projects due to its safety within the human and murine gut [31]. We modeled how our circuit would behave with different parameter values, informing our choices of engineered elements within the circuit. We varied ribosome binding sites experimentally, showing that the tetrathionate two-component system's stronger response could be recapitulated by varying ribosome binding rates in our model.

Computationally, we designed chemical reaction networks that model our microbial-based circuits in silico. We screened a wide variety of parameters, drawing from previously published datasets. In our sensing system modeling, we found that varying ribosome binding rates to regulator transcripts varies the tetrathionate response. Experimentally, we optimized the sensitivity of the two-component tetrathionate inflammation system in E. coli Nissle. We screened a library containing various RBS strengths and quantified the circuit's sensitivity as a function of fluorescent readout. The engineered strain LM19 demonstrated the largest dynamic range across therapeutically relevant concentrations of tetrathionate. We found experimentally varying RBS strength results in different fold changes consistent with our model.

We incorporated sensor logic by placing inputs of the split activator AND gate under the regulation of the tetrathionate and IPTG response promoters. After experimental tuning to minimize leak, this construct is activated when both tetrathionate and IPTG are present. By creating a chemical reaction network model of our full AND gate, we were able to identify potential causes for leak in our system. Experimentally, these cases may offer interesting pathways for studying protein-based logic gates. As we engineer additional functionality to this circuit, we hope that our in silico RBS tuning and insight into leak will offer us more understanding of our in vitro results.

In summary, our results demonstrate the usefulness of modular synthetic biological parts and circuit components to design circuits in microbial chassis capable of logically combining two independent input signals, one of which is associated with medical applications. We find that logic gates previously described by Wang et al. can be optimized in E. coli Nissle, allowing for future directions in OR, NOT, NOR, and NAND integration. In future work, we will characterize our circuit's ability to sense and respond to medically-induced inflammation and input signal IPTG in vivo. The engineered circuit's functional stability moving from a controlled, in vitro environment to the gut microbiome's competitive environment presents a significant challenge from the competition and metabolic burden perspective. As a continuation, we aim to engineer the second input increase spatial targeting within the gut.

Engineered microbes can deliver effective therapeutics with exquisite spatial and temporal resolution in medically relevant inflammatory conditions. Synthetic biology may offer advantages over traditional chronic inflammation therapies by designing targeted drug delivery to tissues affected by disease and rather than risk off-target effects. The models and logic optimization reported here are a preliminary step towards this long term goal.

\section{Acknowledgements}

We thank William Poole and John Marken for technical guidance, as well as all other Murray lab members for insightful discussions. We thank Dr. Henry Schreiber and Prof. Sarkis Mazmanian for collaborating with us in ongoing microbiome studies with this project. Dr. Leo Green is supported by the Caltech NSF AGEP Fellowship and Rosen Fellowship. We thank Caltech CEMI for providing support for future directions of this work. Prof. Justin Bois has provided excellent discussions regarding data analysis and availability. Logic gate strains were provided by Martin Buck \& Baojun Wan. Some figures were created with BioRender.com. 


\section{References}

1. Schulz, M. D. et al. High-fat-diet-mediated dysbiosis promotes intestinal carcinogenesis independently of obesity. en. Nature 514. Number: 7523 Publisher: Nature Publishing Group, 508-512. ISSN: 1476-4687. https://www .nature.com/articles/nature13398 (2020) (Oct. 2014).

2. Sharon, G., Sampson, T. R., Geschwind, D. H. \& Mazmanian, S. K. The Central Nervous System and the Gut Microbiome. Cell 167, 915-932. ISSN: 0092-8674. https://www.ncbi.nlm.nih.gov/pmc/ articles/PMC5127403/ (2020) (Nov. 2016).

3. Kau, A. L., Ahern, P. P., Griffin, N. W., Goodman, A. L. \& Gordon, J. I. Human nutrition, the gut microbiome and the immune system. en. Nature 474. Number: 7351 Publisher: Nature Publishing Group, 327-336. ISSN: 1476-4687. https : //www . nature.com/articles/nature10213 (2020) (June 2011).

4. Saeidi, N. et al. Engineering microbes to sense and eradicate Pseudomonas aeruginosa, a human pathogen. Molecular Systems Biology 7. Publisher: John Wiley \& Sons, Ltd, 521. ISSN: 1744-4292. https://www.embopress.org/doi/full/10.1038/msb.2011.55 (2020) (Jan. 2011).

5. Gurbatri, C. R. et al. Engineered probiotics for local tumor delivery of checkpoint blockade nanobodies. en. Science Translational Medicine 12. Publisher: American Association for the Advancement of Science Section: Research Article. ISSN: 1946-6234, 1946-6242. https ://stm. sciencemag.org/content/12/ 530/eaax0876 (2020) (Feb. 2020).

6. Daeffler, K. N.-M. et al. Engineering bacterial thiosulfate and tetrathionate sensors for detecting gut inflammation. Molecular Systems Biology 13. Publisher: John Wiley \& Sons, Ltd, 923. IssN: 1744-4292. https://www.embopress.org/doi/full/10.15252/msb.20167416 (2020) (Apr. 2017).

7. Zeng, M. Y., Inohara, N. \& Nuñez, G. Mechanisms of inflammation-driven bacterial dysbiosis in the gut. eng. Mucosal Immunology 10, 18-26. ISSN: 1935-3456 (2017).

8. Loftus, E. V. Clinical epidemiology of inflammatory bowel disease: incidence, prevalence, and environmental influences. English. Gastroenterology 126. Publisher: Elsevier, 1504-1517. ISSN: 0016-5085, 1528-0012. https://www . gastrojournal .org/article/S0016-5085(04) 00462-7/abstract (2020) (May 2004).

9. Sun, Y. et al. The gut microbiota heterogeneity and assembly changes associated with the IBD. en. Scientific Reports 9. Number: 1 Publisher: Nature Publishing Group, 440. Issn: 2045-2322. https : //www.nature.com/articles/s41598-018-37143-z (2020) (Jan. 2019).

10. Hazel, K. \& O'Connor, A. Emerging treatments for inflammatory bowel disease. Therapeutic Advances in Chronic Disease 11. ISSN: 2040-6223. https://www.ncbi.nlm.nih.gov/pmc/articles/ PMC7003169/ (2020) (Feb. 2020).

11. Chan, W., Chen, A., Tiao, D., Selinger, C. \& Leong, R. Medication adherence in inflammatory bowel disease. eng. Intestinal Research 15, 434-445. ISSN: 1598-9100 (Oct. 2017).

12. Zhou, Z. et al. Engineering probiotics as living diagnostics and therapeutics for improving human health. Microbial Cell Factories 19, 56. ISSN: 1475-2859. https://doi.org/10.1186/s12934-020-01318-z (2020) (Mar. 2020).

13. Hanson, M. L. et al. Oral delivery of IL-27 recombinant bacteria attenuates immune colitis in mice. eng. Gastroenterology 146, 210-221.e13. ISSN: 1528-0012 (Jan. 2014).

14. Vandenbroucke, K. et al. Orally administered L. lactis secreting an anti-TNF Nanobody demonstrate efficacy in chronic colitis. eng. Mucosal Immunology 3, 49-56. ISSN: 1935-3456 (Jan. 2010).

15. Archer, E. J., Robinson, A. B. \& Süel, G. M. Engineered E. coli That Detect and Respond to Gut Inflammation through Nitric Oxide Sensing. ACS Synthetic Biology 1. Publisher: American Chemical Society, 451-457. https://doi.org/10.1021/sb3000595 (2020) (Oct. 2012).

16. Riglar, D. T. et al. Engineered bacteria function in the mammalian gut as long term live diagnostics of inflammation. Nature biotechnology 35, 653-658. ISSN: 1087-0156. https://www.ncbi.nlm.nih.gov/ pmc/articles/PMC5658125/ (2020) (July 2017). 
17. Palmer, J. D. et al. Engineered Probiotic for the Inhibition of Salmonella via Tetrathionate-Induced Production of Microcin H47. ACS Infectious Diseases 4. Publisher: American Chemical Society, 39-45. https://doi.org/10.1021/acsinfecdis.7b00114 (2020) (Jan. 2018).

18. Winter, S. E. et al. Gut inflammation provides a respiratory electron acceptor for Salmonella. eng. Nature 467, 426-429. ISSN: 1476-4687 (Sept. 2010).

19. Wang, B., Kitney, R. I., Joly, N. \& Buck, M. Engineering modular and orthogonal genetic logic gates for robust digital-like synthetic biology. en. Nature Communications 2. Number: 1 Publisher: Nature Publishing Group, 508. ISSN: 2041-1723. https : //www . nature.com/articles/ncomms1516 (2020) (Oct. 2011).

20. Danson, A. E., Jovanovic, M., Buck, M. \& Zhang, X. Mechanisms of $\sigma 54$-Dependent Transcription Initiation and Regulation. en. Journal of Molecular Biology. RNA polymerase reaches 60 431, 39603974. ISSN: 0022-2836. http://www. sciencedirect.com/science/article/pii/S0022283619302293 (2020) (Sept. 2019).

21. Hoch, J. A. Two-component and phosphorelay signal transduction. eng. Current Opinion in Microbiology 3, 165-170. ISSN: 1369-5274 (Apr. 2000).

22. Anderson, C. J. Ribosome Binding Sites/Prokaryotic/Constitutive/Anderson 2010. https://parts . igem.org/Ribosome\%7B\%5C_\%7DBinding\%7B\%5C_\%7DSites/Prokaryotic/Constitutive/Anderson.

23. Kremling, A., Heermann, R., Centler, F., Jung, K. \& Gilles, E. D. Analysis of two-component signal transduction by mathematical modeling using the KdpD/KdpE system of Escherichia coli. en. Biosystems 78, 23-37. ISSN: 0303-2647. http://www.sciencedirect.com/science/article/pii/ S0303264704001145 (2020) (Dec. 2004).

24. Poole, W., Pandey, A., Tuza, Z., Shur, A. \& Murray, R. M. BioCRNpyler: Compiling Chemical Reaction Networks from Biomolecular Parts in Diverse Contexts. bioRxiv (2020).

25. Swaminathan, A., Poole, W., Hsiao, V. \& Murray, R. M. Fast and flexible simulation and parameter estimation for synthetic biology using bioscrape. bioRxiv, 121152 (2019).

26. Merk, L. Logical Inflammation Modeling. https://github.com/lianamerk/Logical_Inflammation/ tree/master.

27. Halleran, A. D., Swaminathan, A. \& Murray, R. M. Single Day Construction of Multigene Circuits with 3G Assembly. ACS Synthetic Biology 7. Publisher: American Chemical Society, 1477-1480. https: //doi.org/10.1021/acssynbio.8b00060 (2020) (May 2018).

28. Espah Borujeni, A. et al. Precise quantification of translation inhibition by mRNA structures that overlap with the ribosomal footprint in N-terminal coding sequences. en. Nucleic Acids Research 45. Publisher: Oxford Academic, 5437-5448. ISSN: 0305-1048. https://academic.oup.com/nar/article/ 45/9/5437/2965383 (2020) (May 2017).

29. Meyer, A. J., Segall-Shapiro, T. H., Glassey, E., Zhang, J. \& Voigt, C. A. Escherichia coli "Marionette" strains with 12 highly optimized small-molecule sensors. eng. Nature Chemical Biology 15, 196-204. ISSN: 1552-4469 (2019).

30. Poole, W., Pandey, A., Shur, A., Tuza, Z. A. \& Murray, R. M. BioCRNpyler: Compiling Chemical Reaction Networks from Biomolecular Parts in Diverse Contexts. bioRxiv. eprint: https : / / www . biorxiv.org/content/early/2020/08/03/2020.08.02.233478.full.pdf. https://www. biorxiv . org/content/early/2020/08/03/2020.08.02.233478 (2020).

31. Crook, N. et al. Adaptive Strategies of the Candidate Probiotic E. coli Nissle in the Mammalian Gut. en. Cell Host 83 Microbe 25, 499-512.e8. ISSN: 1931-3128. http://www.sciencedirect.com/science/ article/pii/S1931312819301015 (2020) (Apr. 2019).

32. Hoyle, R. B., Avitabile, D. \& Kierzek, A. M. Equation-free analysis of two-component system signalling model reveals the emergence of co-existing phenotypes in the absence of multistationarity. PLoS Comput Biol 8, e1002396 (2012). 
33. Dayton, C. J., Prosen, D. E., Parker, K. L. \& Cech, C. L. Kinetic measurements of Escherichia coli RNA polymerase association with bacteriophage T7 early promoters. en. Journal of Biological Chemistry 259. Publisher: American Society for Biochemistry and Molecular Biology, 1616-1621. ISSN: 0021-9258, 1083351X. http://www.jbc.org/content/259/3/1616 (2020) (Feb. 1984).

34. Hoops, S. et al. COPASI - a complex pathway simulator. Bioinformatics 22, 3067-3074 (2006).

35. Der, B. S. et al. DNAplotlib: Programmable Visualization of Genetic Designs and Associated Data. ACS Synthetic Biology 6. Publisher: American Chemical Society, 1115-1119. https://doi.org/10. 1021/acssynbio.6b00252 (2020) (July 2017). 


\section{Supplementary Information}

In this section, we describe the two-component system model in detail. The notation $x: y$ denotes a complex between the chemical species $x$ and $y$. Moreover, all binding rates are denoted with a "b" superscript, and unbinding reactions are denoted with a " $\mathrm{u}$ " superscript. A superscript $P$ denotes phosphorylated species and an asterisk superscript is used to denote activated form of a species. The model description is given in Table 1 and the parameter values can be found in Table 2. All code to regenerate the simulations is publicly available on Github [26].

Table 1: The Two-Component System Model

\begin{tabular}{|c|c|}
\hline Description & Reaction \\
\hline \multicolumn{2}{|c|}{ Transcription and Translation of Regulators } \\
\hline RNA Polymerase binds to P1d & $\mathrm{P}+\mathrm{P} 1 \mathrm{~d} \underset{k_{1}^{u}}{\stackrel{k_{1}^{b}}{\rightleftharpoons}} \mathrm{P} 1 \mathrm{~d}: \mathrm{P}$ \\
\hline Transcription of ttrS, ttrR & $\mathrm{P} 1 \mathrm{~d}: \mathrm{P} \stackrel{k_{t x}}{\longrightarrow} \operatorname{ttr} \mathrm{S}_{\mathrm{T}}+\operatorname{ttrR}_{\mathrm{T}}+\mathrm{P} 1 \mathrm{~d}+\mathrm{P}$ \\
\hline Translation $\operatorname{ttrS}_{\mathrm{T}}$ to $\operatorname{ttrS}$ & $\operatorname{ttrS}_{\mathrm{T}}+\mathrm{R} \underset{k_{2}^{u}}{\stackrel{k_{2}^{b}}{\rightleftharpoons}} \operatorname{ttrS}_{\mathrm{T}}: \mathrm{R} \stackrel{k_{t l}}{\longrightarrow} \operatorname{ttrS}_{\mathrm{T}}+\mathrm{R}+\mathrm{ttrS}$ \\
\hline Translation $\operatorname{ttrR}_{\mathrm{T}}$ to $\operatorname{ttrR}$ & $\operatorname{ttrR}_{\mathrm{T}}+\mathrm{R} \underset{k_{3}^{u}}{\stackrel{k_{3}^{b}}{\rightleftharpoons}} \operatorname{ttr} \mathrm{R}_{\mathrm{T}}: \mathrm{R} \stackrel{k_{t l}}{\longrightarrow} \operatorname{ttrR}_{\mathrm{T}}+\mathrm{R}+\operatorname{ttrR}$ \\
\hline \multicolumn{2}{|c|}{ Tetrathionate Regulator Phosphorylation Pathway } \\
\hline Tetrathionate (tt) triggering ttrS phosphorylation & $\mathrm{ttrS}+\mathrm{tt} \underset{k_{4}^{u}}{\stackrel{k_{4}^{b}}{\rightleftharpoons}} \operatorname{ttrS}^{P}+\mathrm{tt}$ \\
\hline ttrR binding to unphosphorylated ttrS & $\operatorname{ttrR}+\operatorname{ttrS} \underset{k_{5}^{u}}{\stackrel{k_{5}^{b}}{\rightleftharpoons}} \operatorname{ttrR}: \operatorname{ttrS}$ \\
\hline ttrR binding to phosphorylated ttrS & $\operatorname{ttrR}+\operatorname{ttrS}^{P} \underset{k_{6}^{u}}{\stackrel{k_{6}^{b}}{\rightleftharpoons}} \operatorname{ttrR}: \operatorname{ttrS}{ }^{P}$ \\
\hline Phosphorylation of ttrR & $\operatorname{ttrR}: \operatorname{ttrS} P \underset{k_{7}^{u}}{\stackrel{k_{7}^{b}}{\rightleftharpoons}} \operatorname{ttrR}^{P}+\operatorname{ttrS}$ \\
\hline Dephosphorylation of ttrR & $\operatorname{ttrR}^{P} \stackrel{k_{\text {dephos }}}{\longrightarrow} \operatorname{ttrR}$ \\
\hline \multicolumn{2}{|c|}{ Response Regulator Gene Activation } \\
\hline Dimerization of $\operatorname{ttrR}^{P}$ & $\operatorname{ttrR}^{P}+\operatorname{ttrR}^{P} \underset{k_{8}^{u}}{\stackrel{k_{8}^{b}}{\rightleftharpoons}} \operatorname{ttrR}_{2}^{P}$ \\
\hline $\operatorname{ttrR}^{P}$ dimer binding to $\mathrm{pTtr}$ promoter & $\operatorname{ttrR}^{P}+\mathrm{pTtr} \underset{k_{9}^{u}}{\stackrel{k_{9}^{b}}{\rightleftharpoons}} \mathrm{pTtr}^{*}$ \\
\hline RNA Polymerase binds to pTtr* & $\mathrm{P}+\mathrm{pTtr}^{*} \underset{k_{10}^{u}}{\stackrel{k_{10}^{b}}{\rightleftharpoons}} \mathrm{p} \operatorname{Ttr}^{*}: \mathrm{P}$ \\
\hline GFP Transcription & $\mathrm{pTtr}^{*}: \mathrm{P} \stackrel{k_{t x}}{\longrightarrow} \mathrm{pTtr}^{*}+\mathrm{P}+\mathrm{GFP}_{\mathrm{T}}$ \\
\hline GFP Translation & $\mathrm{GFP}_{\mathrm{T}}+\mathrm{R} \underset{k_{11}^{u}}{\stackrel{k_{11}^{b}}{\rightleftharpoons}} \mathrm{GFP}_{\mathrm{T}}: \mathrm{R} \stackrel{k_{t l}}{\longrightarrow} \mathrm{GFP}_{\mathrm{T}}+\mathrm{R}+\mathrm{GFP}$ \\
\hline \multicolumn{2}{|c|}{ Degradation Reactions } \\
\hline $\begin{array}{l}\operatorname{ttrS}_{\mathrm{T}} \text { and ttrS degradation } \\
\operatorname{ttrR}_{\mathrm{T}} \text { and ttrR degradation } \\
\mathrm{GFP}_{\mathrm{T}} \text { and } \mathrm{GFP} \text { degradation }\end{array}$ & $\begin{array}{ll}\operatorname{ttrS}_{\mathrm{T}} \stackrel{\delta}{\rightarrow} \varnothing & \operatorname{ttrS} \stackrel{\delta}{\rightarrow} \varnothing \\
\operatorname{ttrR}_{\mathrm{T}} \stackrel{\delta}{\rightarrow} \varnothing & \operatorname{ttrR} \stackrel{\delta}{\rightarrow} \varnothing \\
\mathrm{GFP}_{\mathrm{T}} \stackrel{\delta}{\rightarrow} \varnothing & \mathrm{GFP} \stackrel{\delta}{\rightarrow} \varnothing\end{array}$ \\
\hline
\end{tabular}


Table 2: Model parameters

\begin{tabular}{|c|c|c|c|c|}
\hline S.no. & Parameters & Description & Guess & Reference \\
\hline 1 & $k_{t x}$ & Transcription rate & 0.1 transcripts $/$ second & Guess \\
\hline 2 & $k_{t l}$ & Translation rate & 1 & Guess \\
\hline 3 & $k_{\text {dephos }}$ & Dephosphorylation rate & 50 & {$[32]$} \\
\hline 4 & $k_{1}^{b}$ & Binding of RNA polymerase to P1d & 10 & {$[33]$} \\
\hline 5 & $k_{1}^{u}$ & $\begin{array}{l}\text { Unbinding of RNA polymerase } \\
\text { and P1d complex }\end{array}$ & 0.0001 & {$[33]$} \\
\hline 6 & $k_{2}^{b}$ & Binding of ttrS transcript to its ribosome & 0.3 & {$[32]$} \\
\hline 7 & $k_{2}^{u}$ & Unbinding of ttrS and ribosome complex & Varies & Guess \\
\hline 8 & $k_{3}^{b}$ & Binding of ttrR transcript to its ribosome & 0.3 & {$[32]$} \\
\hline 9 & $\stackrel{s}{k_{3}^{u}}$ & Unbinding of ttrR and ribosome complex & Varies & Guess \\
\hline 10 & $k_{4}^{b}$ & Binding of tetrathionate to ttrS & 1.6 & {$[32]$} \\
\hline 11 & $k_{4}^{u}$ & Unbinding of tetrathionate and ttrS complex & 0.016 & {$[32]$} \\
\hline 12 & $k_{5}^{b}$ & Binding of ttrR to ttrS & 0.0001 & {$[32]$} \\
\hline 13 & $k_{5}^{u}$ & Unbinding of ttrR and ttrS complex & 6 & Guess \\
\hline 14 & $k_{6}^{b}$ & Binding of ttrR to phosphorylated ttrS & 0.0001 & {$[32]$} \\
\hline 15 & $k_{6}^{u}$ & Unbinding of $\operatorname{ttrR}$ and $\operatorname{ttrS} \mathrm{S}^{\mathrm{P}}$ complex & 1 & Guess \\
\hline 16 & $k_{7}^{b}$ & Forward rate for phosphorylation of ttrR & 1 & {$[32]$} \\
\hline 17 & $k_{7}^{u}$ & Reverse rate for phosphorylation of ttrR & 1 & {$[32]$} \\
\hline 18 & $k_{8}^{b}$ & Dimerization rate of phosphorylated ttrR & 0.0083 & {$[32]$} \\
\hline 19 & $k_{8}^{u}$ & $\begin{array}{l}\text { Unbinding of the dimerized complex } \\
\text { of phosphorylated ttrR }\end{array}$ & 0.5 & [32] \\
\hline 20 & $k_{9}^{b}$ & Forward rate of activation of pTtr promoter & 0.3 & {$[32]$} \\
\hline 21 & $k_{9}^{u}$ & Reverse rate of activation of pTtr promoter & 0.0001 & Guess \\
\hline 22 & $k_{10}^{b}$ & $\begin{array}{l}\text { Binding of RNA polymerase to } \\
\text { activated pTtr promoter }\end{array}$ & 10 & {$[33]$} \\
\hline 23 & $k_{10}^{u}$ & $\begin{array}{l}\text { Unbinding of RNA polymerase and } \\
\text { activated pTtr promoter complex }\end{array}$ & 0.0001 & {$[33]$} \\
\hline 24 & $k_{11}^{b}$ & Binding of GFP transcript to its ribosome & 100 & Guess \\
\hline 25 & $k_{11}^{u}$ & $\begin{array}{l}\text { Unbinding of GFP transcript } \\
\text { and ribosome complex }\end{array}$ & 10 & Guess \\
\hline
\end{tabular}

\section{Sensitivity Analysis of Two-Component System Model}

We performed sensitivity analysis of the two-component system model that describes the tetrathionate sensor circuit. We looked at the sensitivity of the output GFP fluorescence to changes in reaction rate parameters. We used COPASI [34], an analysis software for biological models, to compute the normalized sensitivity coefficients of GFP with different parameter values. A sensitivity coefficient is given as the partial derivative of the output for each parameter,

$$
S=\frac{\partial[\mathrm{GFP}]}{\partial \theta},
$$

where $\theta$ is any model parameter. The COPASI sensitivity analysis tools compute this by using the finite difference numerical approximation. We plot the sensitivity analysis results at the end of the time-course to show the effects of each parameter on the steady-state reporter protein level.

From the results shown in Figure 7, we can see that some of the sensitive parameters are the ribosomebinding and unbinding parameters. Although there are other sensitive parameters in the model, they are not all tunable. This simple model-based analysis confirms our hypothesis and results presented in Figure 2, where we tuned the ribosome binding strength to optimize the two-component sensor performance. 


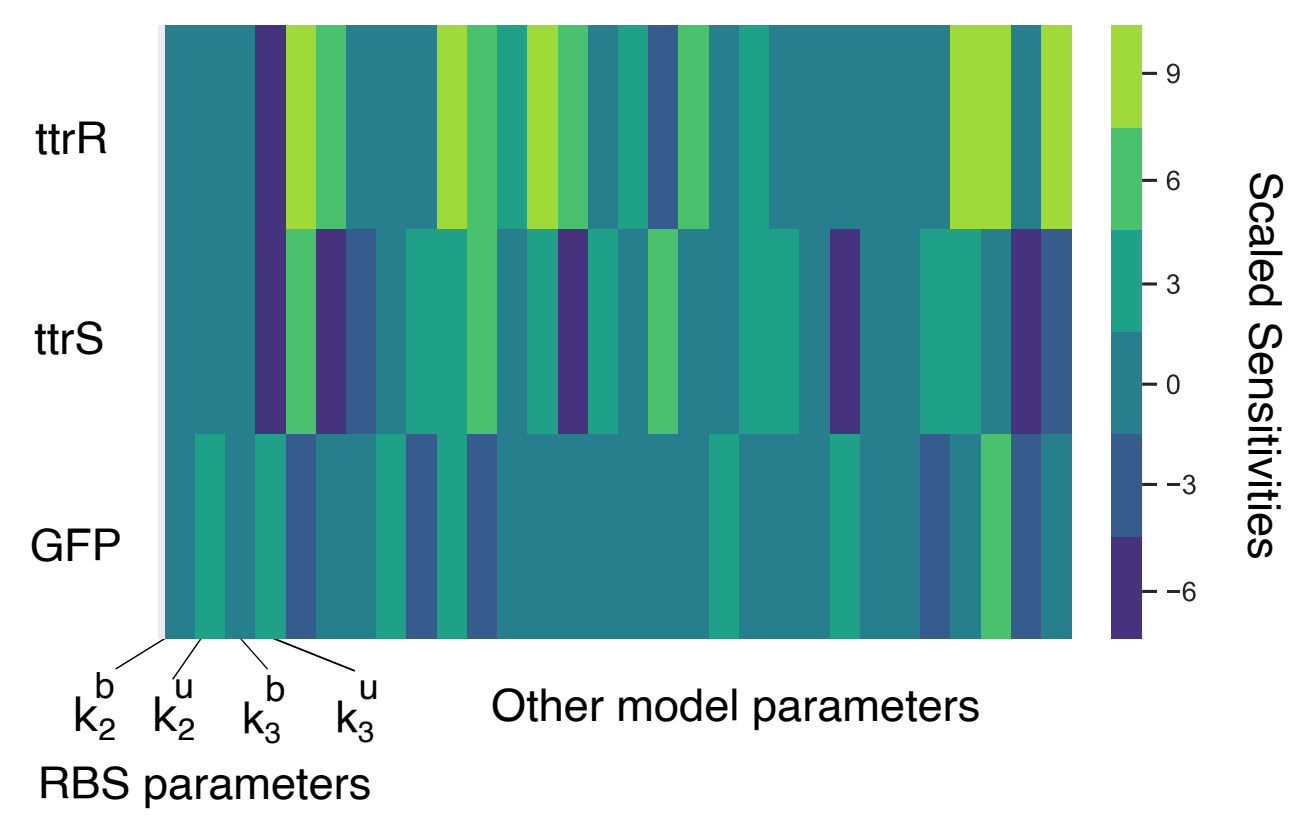

Figure 7: Sensitivity analysis of tetrathionate sensor model. Heatmap denoting the sensitivity of three key species in the tetrathionate sensor model with respect to model parameters. The tunable parameters in the experiment are the ribosome binding strengths that are denoted in the figure.

\section{AND gate constructs}

The detailed AND gate constructs are described in Figure 8. Sequences are available as GenBank files on Github [26]. Circuit diagram plots were created with DNAplotlib [35].

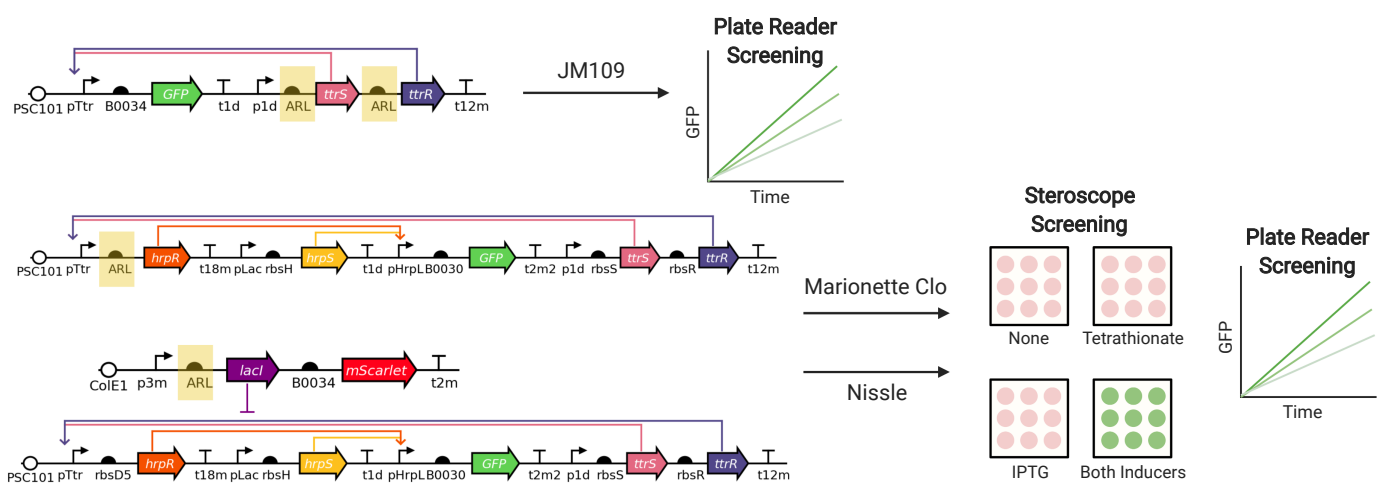

Figure 8: Constructs and Screening Stages. The tetrathionate construct was optimized using plate reader screening, while the AND gates were first screened on inducer plates. Stereoscope images were taken, and colonies that were OFF in all cases except both inducers were then chosen for further plate reader assays. Note in the full AND gate, mScarlet is constitutively expressed, such that the one or no inducer plates are still red fluorescent. 